\title{
SU(1,1)-type light-atom-correlated interferometer
}

\author{
Hongmei Ma, ${ }^{1}$ Dong Li, ${ }^{1}$ Chun-Hua Yuan, ${ }^{1, *}$ L. Q. Chen, ${ }^{1, \dagger}$ Z. Y. Ou, ${ }^{1,2}$ and Weiping Zhang ${ }^{1, \ddagger}$ \\ ${ }^{1}$ Quantum Institute for Light and Atoms, Department of Physics, East China Normal University, Shanghai 200062, People's Republic of China \\ ${ }^{2}$ Department of Physics, Indiana University-Purdue University Indianapolis, 402 North Blackford Street, Indianapolis, Indiana 46202, USA
}

(Received 28 February 2015; published 24 August 2015)

\begin{abstract}
The quantum correlation of light and atomic collective excitation can be used to compose an SU(1,1)-type hybrid light-atom interferometer, where one arm in the optical SU(1,1) interferometer is replaced by the atomic collective excitation. The phase-sensing probes include not only the photon field but also the atomic collective excitation inside the interferometer. For a coherent squeezed state as the phase-sensing field, the phase sensitivity can approach the Heisenberg limit under the optimal conditions. We also study the effects of the loss of light field and the dephasing of atomic excitation on the phase sensitivity. This kind of active SU $(1,1)$ interferometer can also be realized in other systems, such as circuit quantum electrodynamics in microwave systems, which provides a different method for basic measurement using the hybrid interferometers.
\end{abstract}

DOI: 10.1103/PhysRevA.92.023847 PACS number(s): 42.50.St, 42.65.Dr, 42.50.Lc, 42.65.Yj

\section{INTRODUCTION}

Enhanced phase estimation is important for high-precision measurements of physical parameters [1-3]. In optical measurements, many physical parameters can be converted to phase measurements of the optical field based on the interferometer. The phase-measurement precision can be described by quantum Fisher information and the quantum Cramér-Rao bound [4,5]. The mean-square error in phase $\phi$ is given by the error-propagation formula, $\delta \phi=\Delta A|d\langle A\rangle / d \phi|^{-1}$, where the average $\langle A\rangle$ and standard deviation $\Delta A=\sqrt{\left\langle A^{2}\right\rangle-\langle A\rangle^{2}}$ of an observable $A$ are calculated in an optimal condition. High precisions based on the interferometer can be reached by reducing the uncertainty $\Delta A$ or increasing the slope $d\langle A\rangle / d \phi$, or operating them at the same time. The squeezed states [6-9] and two-mode squeezed states $[10,11]$ are used to make the noise $\left(\Delta^{2} A\right)$ below vacuum noise. The slope can be improved by the oscillation frequency or the amplitude enhancements of the output signal. Beating the standard quantum limit (SQL) based on the oscillation-frequency enhancement was realized by the NOON states [12,13]. The amplitude improvement of the output signal was realized by changing the structure of the interferometer, where the 50-50 beam splitters (BSs) in a traditional Mach-Zehnder interferometer (MZI) were replaced by the optical parameter amplifiers (OPAs) [14]. This interferometer was introduced by Yurke et al. [14] and is also called the SU(1,1) interferometer because it is described by the $\mathrm{SU}(1,1)$ group, as opposed to $\mathrm{SU}(2)$ for BSs. The $\mathrm{SU}(1,1)$ interferometry is under experimental investigation by different groups $[15,16]$ and even with Bose-Einstein condensates (BECs) [17-20]. Peise et al. [19] exploited the quantum Zeno effects using the method of interaction-free measurements and Gabbrielli et al. [20] realized a nonlinear three-mode interferometer, where the analogy of optical down conversion, i.e., the basic ingredient of SU $(1,1)$ interferometry, is created with ultracold atoms.

\footnotetext{
*chyuan@phy.ecnu.edu.cn

$\dagger$ lqchen@phy.ecnu.edu.cn

${ }^{\ddagger}$ wpzhang@phy.ecnu.edu.cn
}

Recently, an improved theoretical scheme was presented by Plick et al. [21], who proposed to inject a strong coherent beam to "boost" the photon number. Experimental realization of this $\mathrm{SU}(1,1)$ interferometer was reported recently [15]. The maximum output intensity of this interferometer can be much higher than the input intensity as well as the intensity inside the interferometer (the phase-sensing intensity). More recently, the noise performance of this interferometer was analyzed [22,23]. Experimentally, under the same phasesensing intensity condition, the improvement of $4.1 \mathrm{~dB}$ in signal-to-noise ratio of this interferometer over a traditional linear interferometer was observed [24]. Due to the improved phase-measurement sensitivity of this interferometer, it was suggested for gravitational wave detection, but it needs strong coherent light input [21]. The very strong coherent light will generate the higher-order nonlinear effect and the radiation pressure noise. Combined with the squeezed-state input, the sensitivity of $\mathrm{SU}(1,1)$ can be improved further due to the noise reduction [23]. Collective atomic excitation due to its potential applications for quantum information processing has attracted a great deal of interest [25-32]. Here, we present an SU(1,1)-type hybrid interferometer composed of the light and atomic collective excitation. There are two advantages. One is high conversion based on the Raman process. The other, more important one is that the phase shift is from either or both the optical phase and the phase of the atomic collective excitation which is sensitive to magnetic fields due to the Zeeman effect. Such an interferometer should find wide applications in precision measurement in atomic and optical physics. Our scheme presents an extension and may be a substantial step forward in an $\mathrm{SU}(1,1)$ standard optical interferometer.

In the SU(1,1)-type hybrid light-atom-correlated interferometer discussed here, we use a Raman process to produce a Stokes field together with a correlated atomic collective excitation; that is, one arm in the optical SU(1,1) interferometer is replaced by the atomic collective excitation. For a coherent squeezed state as a phase-sensing field input, the phase sensitivity can approach the Heisenberg limit. The effects of photon loss and collisional dephasing of the atomic excitation on the the phase sensitivity are analyzed. 


\section{LIGHT-ATOM-CORRELATED INTERFEROMETER}

Let us first introduce the SU(1,1)-type hybrid light-atomcorrelated interferometer. In the optical SU $(1,1)$ interferometer of Yurke et al. [14], two nonlinear beam splitters take the place of two linear beam splitters in the traditional Mach-Zehnder interferometer (MZI). In the SU(1,1)-type hybrid light-atomcorrelated interferometer, one of two arms in the optical $\mathrm{SU}(1,1)$ interferometer is replaced by an atomic collective excitation, as shown in Fig. 1. In our scheme, the splitting and recombination of the light field and atomic collective excitation are composed of two Raman processes. In the first Raman process, similar to the beam-splitting process, $\hat{b}_{0}$ is in vacuum, i.e., all atoms in their ground hyperfine state $|1\rangle$ by optical pumping, or $\hat{b}_{0}$ is an initial atomic collective excitation which can be prepared by another Raman process [28] or electromagnetically induced transparency process [29]. Then an off-resonant pump light $E_{P 1}$ is applied to the atomic ensemble together with a phase-sensing field $\hat{a}_{0}$, generating stimulating Raman scattering $\hat{a}_{1}$ together with a correlated atomic collective excitation $\hat{b}_{1}[28,30]$. At the same time, the incoming phasesensing field $\hat{a}_{0}$ has been amplified by the Raman process. In the second Raman process, similar to the beam recombination process, the pump field $P_{2}$ together with the generated Stokes field $\hat{a}_{1}$ inject the Raman system again. Before the Stokes field $\hat{a}_{1}$ injects the Raman system, it is subject to phase $\phi$. After they

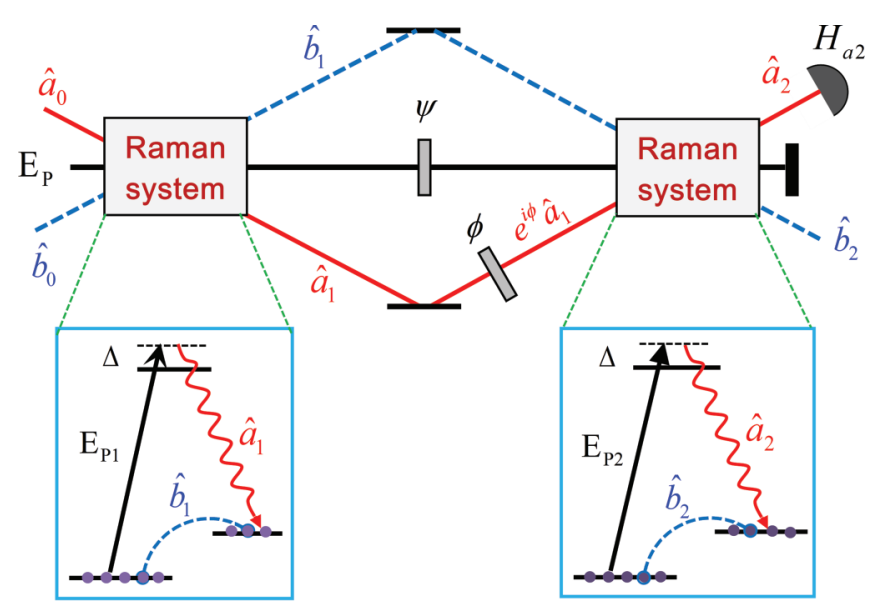

FIG. 1. (Color online) (a) The schematic diagram of the SU(1,1)type light-atom-correlated interferometer. In the optical $\mathrm{SU}(1,1)$ interferometer of Yurke et al., two nonlinear beam splitters take the place of two linear beam splitters in the traditional Mach-Zehnder interferometer (MZI). Here, we use the Raman process (showed in the boxes) to produce a Stokes field $\hat{a}_{i}$ together with a correlated atomic collective excitation $\hat{b}_{i}(i=1,2)$, which are the beam-splitting elements. That is, in the light-atom-correlated interferometer, one arm is the Stokes field (solid line) and the other arm is replaced by the atomic collective excitation (dashed line). Two arms splitting and their recombination are composed of two Raman processes which are successively implemented inside the same atomic system. $\hat{a}_{0}$ is the initial input light field. $\hat{b}_{0}$ is in vacuum or an initial atomic collective excitation which can be prepared by another Raman process or electromagnetically induced transparency process. The pump field $E_{p}$ between the two Raman processes has a $\psi$ phase difference. The output optical mode $\hat{a}_{2}$ is detected by the homodyne detector $H_{a 2} . \phi$ : phase shift. inject the Raman system, the phase-modulated Stokes field $\hat{a}_{2}$ is generated, as shown in Fig. 1 [31,32]. When the phase shift $\phi$ is 0 , the light field and atomic collective excitation are decorrelated by the second Raman process. But when the phase shift $\phi$ is not 0 , similar to the optical interferometer, the phase-measurement sensitivity of this hybrid interferometer is improved due to signal enhancement based on Raman amplification, i.e., the slope of the output signal is improved. Compared with the realization of an $\mathrm{SU}(1,1)$ interferometry via four-wave mixing [15,24], the Raman process has high conversions due to the second-order nonlinear process instead of the third-order nonlinear process. In addition, the proposal can also introduce the atomic phase via a magnetic field change into the phase measurement, and provide a different method for basic measurements in atomic and optical physics.

Next, we analyze the two Raman processes. Considering a three-level $\lambda$-shaped atom system shown in the box of Fig. 1, the Raman-scattering process is described by the following pair of coupled equations [33]:

$$
\frac{\partial \hat{a}(t)}{\partial t}=\eta A_{P} \hat{b}^{\dagger}(t), \frac{\partial \hat{b}(t)}{\partial t}=\eta A_{P} \hat{a}^{\dagger}(t),
$$

where $\hat{a}(t)$ is the light field operator, $\hat{b}(t)$ is the collective atomic operator, $A_{P}$ is the amplitude of the pump field, and $\eta$ is the coupling constant. The solution of the above equation is $\hat{a}(t)=u(t) \hat{a}(0)+v(t) \hat{b}^{\dagger}(0)$ and $\hat{b}(t)=u(t) \hat{b}(0)+v(t) \hat{a}^{\dagger}(0)$, where $u(t)=\cosh (g), v(t)=e^{i \theta} \sinh (g), g=\left|\eta A_{P}\right| t, e^{i \theta}=$ $\left(A_{P} / A_{P}^{*}\right)^{1 / 2}$, and $t$ is the time duration of pump field $E_{P}$. Different initial conditions of $\hat{a}(0)$ and $\hat{b}(0)$ correspond to different scattering processes. We use different subscripts to differentiate the two processes, where 1 denotes the first Raman process (RP1) and 2 denotes the second Raman process (RP2). We first examine the quantum correlations between the two modes $\hat{X}_{a}=\left(\hat{a}+\hat{a}^{\dagger}\right) / 2$ and $\hat{X}_{b}=\left(\hat{b}+\hat{b}^{\dagger}\right) / 2$ of two Raman processes. The correlation of light and atomic collective excitation can be described by the linear correlation coefficient (LCC), which is defined as [34]

$$
J(\hat{X}, \hat{Y})=\frac{\operatorname{cov}(\hat{X}, \hat{Y})}{\left\langle(\Delta \hat{X})^{2}\right\rangle^{1 / 2}\left\langle(\Delta \hat{Y})^{2}\right\rangle^{1 / 2}},
$$

where $\left\langle(\Delta \hat{X})^{2}\right\rangle=\left\langle\hat{X}^{2}\right\rangle-\langle\hat{X}\rangle^{2},\left\langle(\Delta \hat{Y})^{2}\right\rangle=\left\langle\hat{Y}^{2}\right\rangle-\langle\hat{Y}\rangle^{2}$, and $\operatorname{cov}(\hat{X}, \hat{Y})=\frac{1}{2}(\langle\hat{X} \hat{Y}\rangle+\langle\hat{Y} \hat{X}\rangle)-\langle\hat{X}\rangle\langle\hat{Y}\rangle$ is the covariance of the quadrature phase operators $\hat{X}$ and $\hat{Y}$ [35]. For RP1, the LCC $J\left(\hat{X}_{a 1}, \hat{X}_{b 1}\right)$ is dependent on different initial conditions. When $\hat{a}(0)$ and $\hat{b}(0)$ start from the vacuum, the first RP1 created pair correlations. Here the pair is not a photon pair, but is composed of a photon and an atomic collective excitation. The LCC is given by

$$
J\left(\hat{X}_{a 1}, \hat{X}_{b 1}\right)=\cos \theta_{1} \tanh \left(2 g_{1}\right) .
$$

For $\theta_{1}=0\left(A_{P 1}\right.$ is real), the maximum LCC $\operatorname{are} \tanh \left(2 g_{1}\right)$. The generated state is similar to the two-mode squeezed vacuum state $|\Psi\rangle=\sum_{n} c_{n}|n\rangle_{\mathrm{ph}}|n\rangle_{\text {atom }}$, where $n$ is the photon number and the number of atomic collective excitation. If $\hat{a}(0)$ and $\hat{b}(0)$ are initially in coherent states, the generated state is similar to a two-mode squeezed coherent state [36]. After RP1, the LCC is not zero, which shows that a strong correlation exists between the number of photon and the number of atomic collective excitation number. 


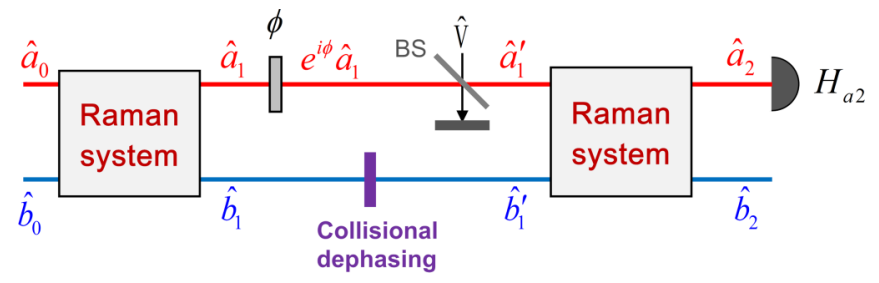

FIG. 2. (Color online) A lossy interferometer model. The loss in the optical arm is modeled by adding a fictitious beam splitter, i.e., $\hat{a}_{1}^{\prime}=\sqrt{T} \hat{a}_{1}\left(t_{1}\right) e^{i \phi}+\sqrt{R} \hat{V}$, where $T$ and $R$ are the transmission and reflectance coefficients, respectively, and $\hat{V}$ is in vacuum. The loss in the other arm is the atomic collisional dephasing, i.e., $\hat{b}_{2}^{\prime}=$ $\hat{b}_{1}\left(t_{1}\right) e^{-\Gamma \tau}+\hat{F}$, where $\Gamma$ is the collisional dephasing rate, $\tau$ is the delay between two Raman processes, and $\hat{F}$ is the noise operator.

Then, we examine the quantum correlation of the RP2. After a delay time $\tau$ of the RP1 generation, the Stokes field $\hat{a}_{1}$ together with the pumping field $E_{P 2}$ drive the atomic system again, shown in Fig. 1. According to the solutions of Eq. (1), we can obtain $\hat{a}_{2}\left(t_{2}\right)=u_{2}\left(t_{2}\right) \hat{a}_{2}(0)+v_{2}\left(t_{2}\right) \hat{b}_{2}^{\dagger}(0)$ and $\hat{b}_{2}\left(t_{2}\right)=$ $u_{2}\left(t_{2}\right) \hat{b}_{2}(0)+v_{2}\left(t_{2}\right) \hat{a}_{2}^{\dagger}(0)$, where $t_{2}$ is the duration of the pump field $E_{P 2} . \hat{a}_{2}(0)$ and $\hat{b}_{2}(0)$ are the initial conditions of RP2, which is from the atomic collective excitation and stokes field of RP1. We consider the collisional dephasing, which can be described by the factor $e^{-\Gamma \tau}$ (see Fig. 2). Then the initial condition $\hat{b}_{2}(0)$ is

$$
\hat{b}_{2}(0)=\hat{b}_{1}\left(t_{1}\right) e^{-\Gamma \tau}+\hat{F},
$$

where $\tau$ is the delay and $\hat{F}=\int_{0}^{\tau} e^{-\Gamma\left(\tau-t^{\prime}\right)} f\left(t^{\prime}\right) d t^{\prime} . \hat{f}(t)$ is the quantum statistical Langevin operator describing the collisioninduced fluctuation and obeys $\left\langle\hat{f}(t) \hat{f}^{\dagger}\left(t^{\prime}\right)\right\rangle=2 \Gamma \delta\left(t-t^{\prime}\right)$ and $\left\langle\hat{f}^{\dagger}(t) \hat{f}\left(t^{\prime}\right)\right\rangle=0$. The Stokes light $\hat{a}_{1}$ is also subject to photon loss, which can be equal to the effect of fictitious beam splitters inserted in the channel, as shown in Fig. 2. Considering the loss in the propagation, the initial condition $\hat{a}_{2}(0)$ is given by

$$
\hat{a}_{2}(0)=\sqrt{T} \hat{a}_{1}\left(t_{1}\right) e^{i \phi}+\sqrt{R} \hat{V},
$$

where $T$ and $R$ are the transmission and reflectance coefficients, respectively, and $\hat{V}$ is in vacuum.

Using the initial conditions given by Eqs. (4) and (5), the generated Stokes field $\hat{a}_{2}$ and atomic collective excitation $\hat{b}_{2}$ can be worked out,

$$
\begin{gathered}
\hat{a}_{2}\left(t_{2}\right)=\mathcal{U}_{1} \hat{a}_{1}(0)+\mathcal{V}_{1} \hat{b}_{1}^{\dagger}(0)+\sqrt{R} u_{2} \hat{V}+v_{2} \hat{F}^{\dagger}, \\
\hat{b}_{2}\left(t_{2}\right)=e^{-i \phi}\left[\mathcal{U}_{2} \hat{b}_{1}(0)+\mathcal{V}_{2} \hat{a}_{1}^{\dagger}(0)\right]+\sqrt{R} v_{2} \hat{V}^{\dagger}+u_{2} \hat{F},
\end{gathered}
$$

where $\mathcal{U}_{1}=\sqrt{T} u_{1} u_{2} e^{i \phi}+e^{-\Gamma \tau} v_{1}^{*} v_{2}, \quad \mathcal{V}_{1}=\sqrt{T} v_{1} u_{2} e^{i \phi}+$ $e^{-\Gamma \tau} u_{1}^{*} v_{2}, \quad \mathcal{U}_{2}=e^{-\Gamma \tau} u_{1} u_{2} e^{i \phi}+\sqrt{T} v_{1}^{*} v_{2}, \quad$ and $\quad \mathcal{V}_{2}=$ $e^{-\Gamma \tau} v_{1} u_{2} e^{i \phi}+\sqrt{T} u_{1}^{*} v_{2}$. When $T=1, \Gamma \tau=0$, it reduced to the ideal lossless case, i.e., $\mathcal{U}_{1}=\mathcal{U}_{2}=\mathcal{U}, \mathcal{V}_{1}=\mathcal{V}_{2}=\mathcal{V}$, and $\mathcal{U}=\left[\cosh g_{1} \cosh g_{2} e^{i\left(\phi+\theta_{1}-\theta_{2}\right)}+\sinh g_{1} \sinh g_{2}\right] e^{i\left(\theta_{2}-\theta_{1}\right)}$, $\mathcal{V}=\left[\sinh g_{1} \cosh g_{2} e^{i\left(\phi+\theta_{1}-\theta_{2}\right)}+\cosh g_{1} \sinh g_{2}\right] e^{i \theta_{2}}$, where $|\mathcal{U}|^{2}-|\mathcal{V}|^{2}=1$. When $\phi=0$ and $\theta_{2}-\theta_{1}=\pi$, we have $\mathcal{U}=1$ and $\mathcal{V}=0$. Therefore, under this condition, the LCC $J\left(\hat{X}_{a 2}, \hat{X}_{b 2}\right)$ is 0 for any input states. That is, the RP2 will "undo" what the RP1 did.

Now, we analyze the phase sensitivity of this lightatom-correlated interferometer. In quantum phase precision measurement, the phase sensitivity $\Delta \phi$ is defined by the linear error propagation,

$$
(\Delta \phi)^{2}=\frac{\left\langle(\Delta \hat{O})^{2}\right\rangle}{|\partial\langle\hat{O}\rangle / \partial \phi|^{2}},
$$

where $\hat{O}$ is the measurable operator and $\left\langle(\Delta \hat{O})^{2}\right\rangle=\left\langle\hat{O}^{2}\right\rangle-$ $\langle\hat{O}\rangle^{2}$. The output amplitude quadrature operator $\hat{X}_{a 2}=\left(\hat{a}_{2}+\right.$ $\left.\hat{a}_{2}^{\dagger}\right) / 2$ is the detected variable. If we use the atomic variable $\hat{X}_{b 2}=\left(\hat{b}_{2}+\hat{b}_{2}^{\dagger}\right) / 2$ as the detected variable, we also get the phase sensitivity of the same order. But the variable $\hat{X}_{b 2}$ needs to read out by another light field. Here, we consider the balanced situation is $g_{1}=g_{2}$, and $\theta_{2}-\theta_{1}=\pi$.

For a coherent light $|\alpha\rangle \quad\left(|\alpha\rangle=\hat{D}(\alpha)|0\rangle, \alpha=|\alpha| e^{i \theta_{\alpha}}\right.$, $\left.N_{\alpha}=|\alpha|^{2}\right)$ and a coherent squeezed state $|\alpha, \zeta\rangle(|\alpha, \zeta\rangle=$ $\hat{D}(\alpha) \hat{S}(\zeta)|0\rangle)$ as the phase-sensing fields, where $\hat{D}(\alpha)=$ $e^{\alpha \hat{a}^{\dagger}-\alpha^{*} \hat{a}}$ and $\hat{S}(\zeta)=e^{\left(\zeta^{*} \hat{a}^{2}-\zeta \hat{a}^{\dagger 2}\right) / 2}, \zeta=r e^{i \theta_{s}}$, and the slopes of the output amplitude quadrature operator $\hat{X}_{a 2}$ are the same and are given by

$$
\left|\frac{\partial\left\langle\hat{X}_{a 2}\right\rangle}{\partial \phi}\right|=\sqrt{T N_{\alpha}} \cosh ^{2} g\left|\sin \left(\phi+\theta_{\alpha}\right)\right| .
$$

When $\phi+\theta_{\alpha}=\pi / 2$, the maximum slope is $\sqrt{T N_{\alpha}} \cosh ^{2} g$. But the output uncertainty is different, for the coherent state and coherent squeezed-state input, and the uncertainties of the output amplitude quadrature operator $\hat{X}_{a 2}$ are given by $\left\langle\left(\Delta \hat{X}_{a 2}\right)^{2}\right\rangle_{c}=\left(\left|\mathcal{U}_{1}\right|^{2}+\left|\mathcal{V}_{1}\right|^{2}\right) / 4+\left[R\left|u_{2}\right|^{2}+\left|v_{2}\right|^{2}\left(1-e^{-2 \Gamma \tau}\right)\right] / 4$ and $\left\langle\left(\Delta \hat{X}_{a 2}\right)^{2}\right\rangle_{s}=\left[\left|\mathcal{U}_{1}\right|^{2}\left(e^{2 r} \sin ^{2} \Theta+e^{-2 r} \cos ^{2} \Theta\right)+\left|\mathcal{V}_{1}\right|^{2}\right] / 4$ $+\left[R\left|u_{2}\right|^{2}+\left|v_{2}\right|^{2}\left(1-e^{-2 \Gamma \tau}\right)\right] / 4$, where $\Theta=\theta_{s} / 2+\theta_{\mathcal{U}_{1}}$ with $\mathcal{U}_{1}=\left|\mathcal{U}_{1}\right| e^{i \theta_{U_{1}}}$, in which the second term $\left[R\left|u_{2}\right|^{2}+\left|v_{2}\right|^{2}\left(1-e^{-2 \Gamma \tau}\right)\right] / 4$ is generated from the loss and collisional dephasing.

The phase sensitivities $(\Delta \phi)_{c}$ and $(\Delta \phi)_{s}$ as a function of the phase-sensing probe number $n_{\mathrm{ph}}$ are shown in Figs. 3(a) and 3(b), respectively. In the presence of the loss and collisional dephasing, the phase sensitivities can beat the SQL under the balanced situation. The phase sensitivity of the coherent squeezed-state input is more easily affected by the loss and collisional dephasing. Figures 4(a) and 4(b) show the phase sensitivity $(\Delta \phi)_{s}$ as a function of the transmission rate $T$ and the collisional dephasing rate $\Gamma \tau$, respectively. Under the condition of $r=2.5, N_{\alpha}=e^{2 r} / 4, g=2$, when $\Gamma \tau \leq 0.3$ or $T \geqslant 0.6$, the phase sensitivity $(\Delta \phi)_{s}$ can beat the SQL. Under the lossless ideal condition $(T=1, \Gamma \tau=0)$, only $(\Delta \phi)_{s}$ can reach the Heisenberg limit (HL). Now, we give the explanations. Under the balanced and lossless situation, the uncertainty of coherent state input is $\left\langle\left(\Delta \hat{X}_{a 2}\right)^{2}\right\rangle_{c}=1 / 4$, which is from the vacuum fluctuation. For coherent squeezed-state input, the uncertainty is $\left\langle\left(\Delta \hat{X}_{a 2}\right)^{2}\right\rangle_{s}=e^{-2 r} / 4$ with $\Theta=0$, which is below the vacuum noise. The reduced noise can improve the phase sensitivity. In the ideal case, the phase sensitivities under the optimal conditions are given by

$$
\begin{aligned}
(\Delta \phi)_{c} & =\frac{1}{\sqrt{N_{\alpha}}} \frac{1}{2 \cosh ^{2} g}, \\
(\Delta \phi)_{s} & =\frac{1}{e^{r} \sqrt{N_{\alpha}}} \frac{1}{2 \cosh ^{2} g},
\end{aligned}
$$



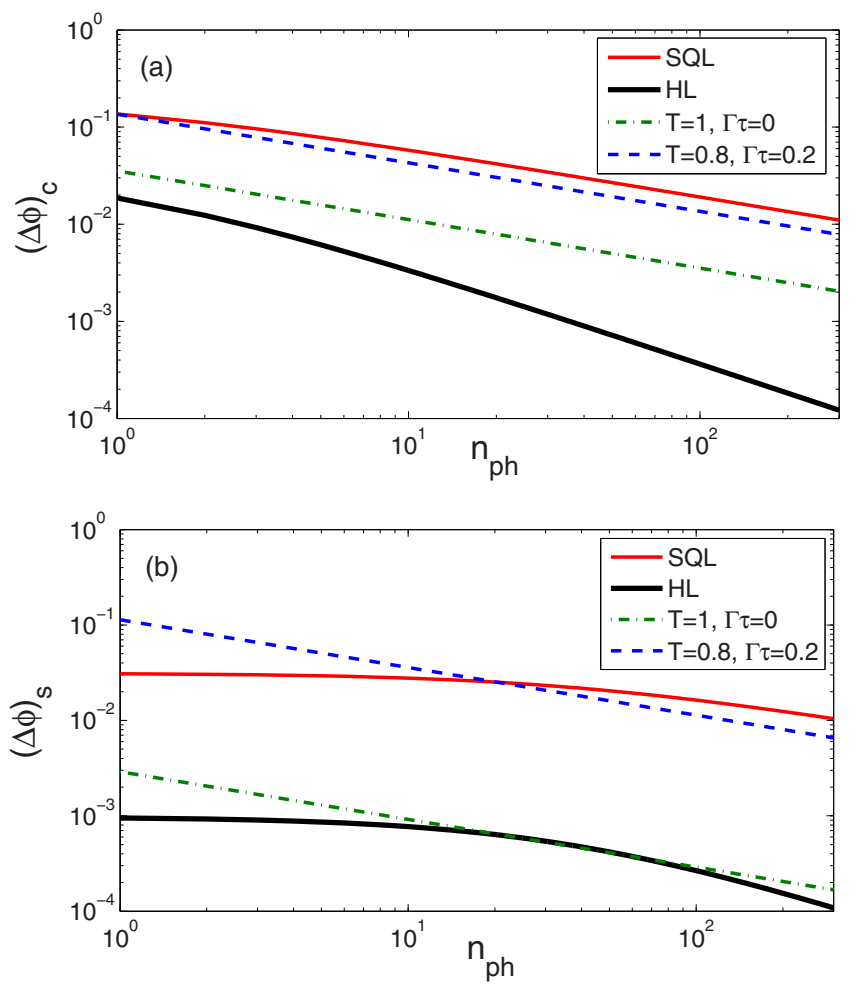

FIG. 3. (Color online) The phase sensitivity (a) $\Delta \phi_{c}$ and (b) $\Delta \phi_{s}$ as a function of the number of probes $n_{\mathrm{ph}}$ inside the interferometer with $g=1$. The input coherent squeezed light with $r=2.5$.

which is improved by $\cosh ^{2} g$ compared to the traditional Mach-Zehnder interferometer for the same input phasesensing field.

Now, we compare the optimal sensitivities given by Eqs. (10) and (11) with SQL $\left(\propto 1 / \sqrt{n_{\mathrm{ph}}}\right)$ and HL $\left(\propto 1 / n_{\mathrm{ph}}\right)$. Here, the phase-sensing field is not the input field as in the traditional MZI, but the amplified field inside the interferometer. Although the phase shift is generated on the light field, the light field and the atomic collective excitation are quantized. The phase-sensing probe number includes not only the photon number $\left\langle\hat{a}_{1}^{\dagger}\left(t_{1}\right) \hat{a}_{1}\left(t_{1}\right)\right\rangle$ but also the atomic collective excitation number $\left\langle\hat{b}_{1}^{\dagger}\left(t_{1}\right) \hat{b}_{1}\left(t_{1}\right)\right\rangle$, which is given by

$$
n_{\mathrm{ph}}=N_{\mathrm{in}}+N_{\mathrm{in}} G_{\mathrm{RP}}+G_{\mathrm{RP}},
$$

where $G_{\mathrm{RP}}=2 \sinh ^{2} g$. The second term $N_{\mathrm{in}} G_{\mathrm{RP}}$ on the righthand side of Eq. (12) is the amplified signal of the input photon number due to the stimulated Raman process, and the last term $G_{\mathrm{RP}}$ is the number of amplified spontaneous-emission photons or noise. For the coherent squeezed-state input case, the input photon number $N_{\text {in }}=\left\langle\alpha, \zeta\left|\hat{a}_{1}^{\dagger}(0) \hat{a}_{1}(0)\right| \alpha, \zeta\right\rangle=|\alpha|^{2}+\sinh ^{2} r$. Under the condition $G_{\mathrm{RP}} \gg 1,|\alpha| \simeq e^{r} / 2 \simeq \sinh r \gg 1$, the total phase-sensing probe number in the interferometer can be written as $n_{\mathrm{ph}} \simeq 2 G_{\mathrm{RP}} N_{\alpha}$. The phase sensitivity of Eq. (11) is given by

$$
(\Delta \phi)_{s} \simeq \frac{1}{2 N_{\alpha}\left(G_{\mathrm{RP}}+2\right)} \approx \frac{1}{n_{\mathrm{ph}}} .
$$

From Eq. (13), with coherent squeezed state as input, the phase sensitivity can reach HL due to the noise reduction and phase-sensing field amplification. As has been previously
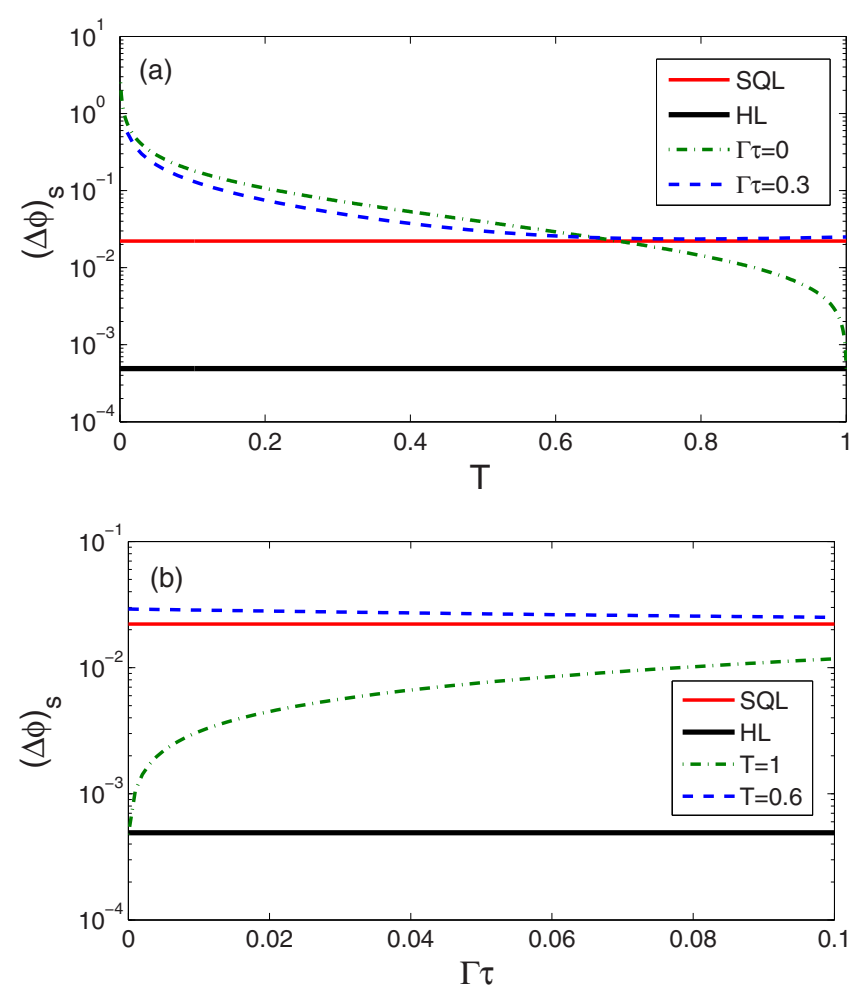

FIG. 4. (Color online) The phase sensitivity $(\Delta \phi)_{s}$ as a function of (a) the transmission rate $T$ and (b) the collisional dephasing rate $\Gamma \tau$ with $r=2.5, N_{\alpha}=e^{2 r} / 4, g=2$.

pointed out, the loss is the limiting factor in precision measurement [22,37-40]. When the transmission $T$ is close to 1 and the collisional dephasing rate $\Gamma \tau$ is very small, the sensitivity is very high and can approach the HL for the coherent squeezed-state input case, as shown in Fig. 4. Enhanced Raman scattering can be obtained by the initially prepared atomic spin excitation $[28,30]$ or by injecting a seeded light field which is correlated with the initially prepared atomic spin excitation [31,32]. This scheme is established on the basis of previous studies and can be realized with high conversions. For Raman scattering, the number of atoms must be bigger than the generated photon number, which is easily realized for hot atoms.

\section{CONCLUSION}

In conclusion, the correlation between light and atomic collective excitation can form an SU(1,1)-type hybrid lightatom-correlated interferometer. The sensitivity is improved due to the signal enhancement compared to the traditional MZI. When the transmission $T$ is close to 1 and the collisional dephasing rate $\Gamma \tau$ is very small, the sensitivity of the coherent squeezed-state input can approach the HL under the optimal condition. This SU(1,1)-type hybrid light-atomcorrelated interferometer can generalize to other systems, such as circuit quantum electrodynamics [41], which provides a different method for basic measurement using the hybrid interferometers. The scheme can be implemented with current technology. 


\section{ACKNOWLEDGMENTS}

This work is supported by the National Basic Research Program of China (973 Program) under Grant No. 2011CB921604, the National NSFC (Grants No.
11474095, No. 11274118, No. 11129402, and No. 11234003), the Innovation Program of Shanghai $\mathrm{Mu}-$ nicipal Education Commission (Grant No. 13ZZ036), and the Fundamental Research Funds for the Central Universities.
[1] V. Giovannetti, S. Lloyd, and L. Maccone, Quantum-enhanced measurements: Beating the standard quantum limit, Science 306, 1330 (2004); Advances in quantum metrology, Nat. Photon. 5, 222 (2011).

[2] R. Schnabel, N. Mavalvala, D. E. McClelland, and P. K. Lam, Quantum metrology for gravitational wave astronomy, Nat. Commun. 1, 121 (2010).

[3] V. Giovannetti, S. Lloyd, and L. Maccone, Quantum Metrology, Phys. Rev. Lett. 96, 010401 (2006).

[4] C. W. Helstrom, Quantum Detection and Estimation Theory (Academic, New York, 1976).

[5] A. S. Holevo, Probabilistic and Statistical Aspect of Quantum Theory (North-Holland, Amsterdam, 1982).

[6] C. M. Caves, Quantum-mechanical noise in an interferometer, Phys. Rev. D 23, 1693 (1981).

[7] M. Xiao, L. A. Wu, and H. J. Kimble, Precision Measurement Beyond the Shot Noise Limit, Phys. Rev. Lett. 59, 278 (1987).

[8] P. Grangier, R. E. Slusher, B. Yurke, and A. LaPorta, SqueezedLight-Enhanced Polarization Interferometer, Phys. Rev. Lett. 59, 2153 (1987).

[9] L. Pezzé and A. Smerzi, Mach-Zehnder Interferometry at the Heisenberg Limit with Coherent and Squeezed-Vacuum Light, Phys. Rev. Lett. 100, 073601 (2008).

[10] G. M. D'Ariano and M. F. Sacchi, Two-mode heterodyne phase detection, Phys. Rev. A 52, R4309 (1995).

[11] O. Steuernagel and S. Scheel, Approaching the Heisenberg limit with two-mode squeezed states, J. Opt. B: Quantum Semiclass. Opt. 6, S66 (2004).

[12] J. P. Dowling, Quantum optical metrology - the lowdown on high-N00N states, Contemp. Phys. 49, 125 (2008).

[13] A. N. Boto, P. Kok, D. S. Abrams,S. L. Braunstein, C. P. Williams, and J. P. Dowling, Quantum Interferometric Optical Lithography: Exploiting Entanglement to Beat the Diffraction Limit, Phys. Rev. Lett. 85, 2733 (2000).

[14] B. Yurke, S. L. McCall, and J. R. Klauder, SU(2) and SU(1 1) interferometers, Phys. Rev. A 33, 4033 (1986).

[15] J. T. Jing, C. J. Liu, Z. F. Zhou, Z. Y. Ou, and W. P. Zhang, Realization of a Nonlinear Interferometer with Parametric Amplifiers, Appl. Phys. Lett. 99, 011110 (2011).

[16] T. S. Horrom, B. E. Anderson, P. Gupta, and P. Lett, SU(1,1) interferometry via four-wave mixing in $\mathrm{Rb}$ (unpublished).

[17] C. Gross, T. Zibold, E. Nicklas, J. Estève, and M. K. Oberthaler, Nonlinear atom interferometer surpasses classical precision limit, Nature (London) 464, 1165 (2010).

[18] Daniel Linnemann, Realization of an SU(1,1) Interferometer with Spinor Bose-Einstein Condensates, Master's thesis, University of Heidelberg, 2013.

[19] J. Peise B. Lücke, L. Pezzé, F. Deuretzbacher, W. Ertmer, J. Arlt, A. Smerzi, L. Santos, and C. Klempt, Interaction-free measurements by quantum Zeno stabilization of ultracold atoms, Nat. Commun. 6, 6811 (2015).
[20] M. Gabbrielli, L. Pezzé, and A. Smerzi, Spin-mixing interferometry with Bose-Einstein condensates, arXiv:1503.08582

[21] W. N. Plick, J. P. Dowling, and G. S. Agarwal, Coherent-lightboosted sub-shot noise quantum interferometry, New J. Phys. 12, 083014 (2010).

[22] Z. Y. Ou, Enhancement of the phase-measurement sensitivity beyond the standard quantum limit by a nonlinear interferometer, Phys. Rev. A 85, 023815 (2012).

[23] D. Li, C. H. Yuan, Z. Y. Ou, and W. P. Zhang, The phase sensitivity of an $\mathrm{SU}(1,1)$ interferometer with coherent and squeezed-vacuum light, New J. Phys. 16, 073020 (2014).

[24] F. Hudelist, J. Kong, C. J. Liu, J. T. Jing, Z. Y. Ou, and W. P. Zhang, Quantum metrology with parametric amplifier based photon correlation interferometers, Nat. Commun. 5, 3049 (2014).

[25] L.-M. Duan, M. D. Lukin, J. I. Cirac, and P. Zoller, Longdistance quantum communication with atomic ensembles and linear optics, Nature (London) 414, 413 (2001).

[26] K. Hammerer, A. S. Sørensen, and E. S. Polzik, Quantum interface between light and atomic ensembles, Rev. Mod. Phys. 82, 1041 (2010).

[27] L. Li, Y. O. Dudin, and A. Kuzmich, Entanglement between light and an optical atomic excitation, Nature (London) 498, 466 (2013).

[28] L. Q. Chen, G.-W. Zhang, C.-H. Yuan, J. Jing, Z. Y. Ou, and W. Zhang, Enhanced Raman Scattering by Spatially Distributed Atomic Coherence, Appl. Phys. Lett. 95, 041115 (2009); L. Q. Chen, G.-W. Zhang, C.-1. Bian, C.-H. Yuan, Z. Y. $\mathrm{Ou}$, and W. Zhang, Observation of the Rabi Oscillation of Light Driven by an Atomic Spin Wave, Phys. Rev. Lett. 105, 133603 (2010).

[29] M. Jain, H. Xia, G. Y. Yin, A. J. Merriam, and S. E. Harris, Efficient Nonlinear Frequency Conversion with Maximal Atomic Coherence, Phys. Rev. Lett. 77, 4326 (1996).

[30] C.-H. Yuan, L. Q. Chen, J. Jing, Z. Y. Ou, and W. Zhang, Coherently enhanced Raman scattering in atomic vapor, Phys. Rev. A 82, 013817 (2010).

[31] C.-H. Yuan, L. Q. Chen, Z. Y. Ou, and W. Zhang, Correlationenhanced phase-sensitive Raman scattering in atomic vapors, Phys. Rev. A 87, 053835 (2013).

[32] Bing Chen, C. Qiu, L. Q. Chen, K. Zhang, J. Guo, C.-H. Yuan, Z. Y. Ou, and W. Zhang, Phase Sensitive Raman Process with Correlated Seeds, Appl. Phys. Lett. 106, 111103 (2015).

[33] M. G. Raymer, Quantum state entanglement and readout of collective atomic-ensemble modes and optical wave packets by stimulated Raman scattering, J. Mod. Opt. 51, 1739 (2004).

[34] C. C. Gerry and P. L. Knight, Introductory Quantum Optics (Cambridge University Press, Cambridge, 2005).

[35] D. F. Walls and G. J. Milburn, Quantum Optics (Springer, Berlin, 1994). 
[36] R. Birrittella, A. Gura, and C. C. Gerry, Coherently stimulated parametric down-conversion, phase effects, and quantumoptical interferometry, Phys. Rev. A 91, 053801 (2015).

[37] G. Gilbert, M. Hamrick, and Y. S. Weinstein, Use of maximally entangled N-photon states for practical quantum interferometry, J. Opt. Soc. Am. B 25, 1336 (2008).

[38] T. Ono and H. F. Hofmann, Effects of photon losses on phase estimation near the Heisenberg limit using coherent light and squeezed vacuum, Phys. Rev. A 81, 033819 (2010).
[39] U. Dorner, R. Demkowicz-Dobrzanski, B. J. Smith, J. S. Lundeen, W. Wasilewski, K. Banaszek, and I. A. Walmsley, Optimal Quantum Phase Estimation, Phys. Rev. Lett. 102, 040403 (2009).

[40] A. M. Marino, N. V. Corzo Trejo and P. D. Lett, Effect of losses on the performance of an SU(1,1) interferometer, Phys. Rev. A 86, 023844 (2012).

[41] Sh. Barzanjeh, D. P. DiVincenzo, and B. M. Terhal, Dispersive qubit measurement by interferometry with parametric amplifiers, Phys. Rev. B 90, 134515 (2014). 